\title{
MONOTONICITY OF ZEROS OF ORTHOGONAL LAURENT POLYNOMIALS*
}

\author{
DIMITAR K. DIMITROV ${ }^{\dagger}$ AND A. SRI RANGA
}

\begin{abstract}
Monotonicity of zeros of orthogonal Laurent polynomials associated with a strong distribution with respect to a parameter is discussed. A natural analog of a classical result of A. Markov is proved. Recent results of Ismail and Muldoon based on the Hellman-Feynman theorem are also extended to a monotonicity criterion for zeros of Laurent polynomials. Results concerning the behaviour of extreme zeros of orthogonal Laurent polynomials are proved. The monotonicity of the zeros of Laguerre-Laurent and Jacobi-Laurent polynomials are investigated.
\end{abstract}

1. Introduction and statement of results. The distribution $d \psi(x)$ is said to be a strong distribution in $(a, b) \subset(0, \infty)$ if $\psi(x)$ is a real bounded nondecreasing function on $(a, b)$ with infinitely many points of increase there, and furthermore, all the moments

$$
\mu_{k}=\int_{a}^{b} x^{k} d \psi(x), \quad k=0, \pm 1, \pm 2, \ldots
$$

exist.

For any given strong distribution in $(a, b)$ there exists a unique sequence, up to a nonzero constant factor normalization, of polynomials $\left\{B_{n}\right\}_{0}^{\infty}$ such that $B_{n}$ is a polynomial of precise degree $n$ and $B_{n}$ satisfies the relations

$$
\int_{a}^{b} x^{-n+k} B_{n}(x) d \psi(x)=0, \quad k=0, \ldots, n-1 .
$$

The first systematic study of these polynomials, which may be called orthogonal Laurent polynomials or simply orthogonal L-polynomials was done by Jones, Thron and Waadeland [10] in connection with the so-called strong Stieltjes moment problem.

Many interesting properties of orthogonal Laurent polynomials are proven in [10]. For example, if $B_{n}$ are normalized to be monic, i.e. to have leading coefficients one, they satisfy the recurrence relation $B_{-1}=0, B_{0}=1$,

$$
B_{n+1}(x)=\left(x-\beta_{n}\right) B_{n}(x)-\delta_{n} x B_{n-1}(x), \quad n \geq 0,
$$

where $\beta_{n}$ and $\delta_{n}, \quad n=0, \ldots$ are positive.

We are particularly interested in the behaviour of the zeros of $B_{n}$. Their location is similar to the one of orthogonal polynomials. All the zeros of $B_{n}$ are real, distinct and lie in $(a, b)$. Moreover, the zeros of $B_{n}$ and $B_{n-1}$ interlace.

Let, for any $\tau \in(p, q), d \psi(x ; \tau)$ be a strong distribution in $x \in(a, b)$. Thus we obtain a parametric sequence $B_{n}(x ; \tau)$ of orthogonal Laurent polynomials where, for any $\tau \in(p, q)$, the polynomials $B_{n}(x ; \tau)$ are L-orthogonal with respect to $d \psi(x ; \tau)$ :

$$
\int_{a}^{b} x^{-n+k} B_{n}(x ; \tau) d \psi(x ; \tau)=0, \quad k=0, \ldots, n-1 .
$$

\footnotetext{
*Received December 14, 1999; accepted for publication June 15, 2001. Research supported by the Brazilian Science Foundations CNPq under Grants 300645/95-3 and 300341/87-3 and FAPESP under Grants 97/6280-0 and 98/02011-8.

${ }^{\dagger}$ Departamento de Ciências de Computação e Estatística, IBILCE, Universidade Estadual Paulista, 15054-000 São José do Rio Preto, SP, Brazil (dimitrov@dcce.ibilce.unesp.br).

${ }^{\ddagger}$ ranga@dcce.ibilce.unesp.br
} 
The $B_{n}(x ; \tau)$ satisfy a recurrence relation of the form

$$
x B_{n}(x ; \tau)=\alpha_{n}(\tau) B_{n+1}(x ; \tau)+\beta_{n}(\tau) B_{n}(x ; \tau)+\gamma_{n}(\tau) x B_{n-1}(x ; \tau), \quad n \geq 0,
$$

with $B_{-1}(x ; \tau)=0, \quad B_{0}(x ; \tau)=1$, where $\alpha_{n}(\tau), \beta_{n}(\tau)$ and $\gamma_{n}(\tau)$ are positive for any $\tau \in(p, q)$. Here the L-orthogonal polynomials are normalized in such a way that the leading coefficient of $B_{n}(x ; \tau)$ is $\left(\alpha_{0}(\tau) \cdots \alpha_{n-1}(\tau)\right)^{-1}$.

We study the monotonicity of zeros of the parametric L-orthogonal polynomials with respect to the parameter $\tau$. Sufficient conditions so that the zeros of $B_{n}(x ; \tau)$ increase (decrease) when $\tau$ increases are given in terms of the behaviour of the strong distibution and also of the coefficients in the recurrence relation.

The problem of monotonicity of zeros of a parametric sequence of orthogonal polynomials has been of interest since Stieltjes' [15] and A.Markov's [11] fundamental contributions. Chapter 6 of Szegö's book [16] gives a general view of the older results. For the newer concepts on the subject we refer to the survey papers of Ismail [6] and Muldoon [12].

In what follows the zeros of $B_{n}(x ; \tau)$ are denoted by $\zeta_{k}(\tau)$ and are supposed to be arranged in decreasing order, $\zeta_{1}(\tau)>\ldots>\zeta_{n}(\tau)$.

First we give an analog of a theorem concerning monotonicity of zeros of orthogonal polynomials due to Ismail [6] which itself can be considered as an extension of a classical result of A. Markov [11] (see also [16, Theorem 6.12.1]).

THEOREM 1.1. Let $B_{n}(x ; \tau), \quad n=0,1, \ldots$ obey the orthogonal property (1.2) with respect to the strong distribution $d \psi(x ; \tau)$,

$$
d \psi(x ; \tau)=\omega(x ; \tau) d \psi(x),
$$

where $\omega(x ; \tau)$ is positive and has continuous first derivative, with respect to $\tau$, for $x \in(a, b)$ and $\tau \in(p, q)$. Assume that the integrals

$$
\int_{a}^{b} x^{j}(\partial \omega(x ; \tau) / \partial \tau) d \psi(x), \quad j=-n,-n+1, \ldots, 0, \ldots, n-1
$$

converge uniformly on every compact subinterval of $(p, q)$. If

$$
\frac{\partial \ln \omega(x ; \tau)}{\partial \tau}
$$

is an increasing (decreasing) function of $x, \quad x \in(a, b)$, then for any $k, 1 \leq k \leq$ $n, \zeta_{k}(\tau)$ is an increasing (decreasing) function of $\tau$.

The next theorem is an analog of Theorem 2.3 of Ismail and Muldoon [8].

TheOREM 1.2. Let $B_{k}(x ; \tau), \quad k=0,1, \ldots, N$ satisfy (1.3), where $\alpha_{k}(\tau), \beta_{k}(\tau)$ and $\gamma_{k}(\tau), k=0, \ldots, N-1$ are positive and differentiable functions of $\tau, \quad \tau \in(p, q)$. (i) If the matrix

$$
\left(\delta_{i, j-1} \frac{\partial}{\partial \tau} \sqrt{\alpha_{i-1}(\tau) \gamma_{i}(\tau) d_{1}}+\delta_{i, j} \frac{\partial}{\partial \tau} \beta_{i-1}(\tau)+\delta_{i, j+1} \frac{\partial}{\partial \tau} \sqrt{\alpha_{i-2}(\tau) \gamma_{i-1}(\tau) d_{1}}\right)_{i, j=1}^{N},
$$

$\alpha_{-1}(\tau)=\gamma_{N}(\tau)=0$, is positive (negative) definite, then all the zeros of $B_{1}(x ; \tau), \ldots$, $B_{N}(x ; \tau)$, which are smaller than the positive real number $d_{1}$, are strictly increasing 
(decreasing) functions of $\tau, \quad \tau \in(p, q)$.

(ii) If the matrix

$$
\left(\delta_{i, j-1} \frac{\partial}{\partial \tau} \sqrt{\frac{\alpha_{i-1}(\tau) \hat{\gamma}_{i}(\tau)}{d_{2}}}+\delta_{i, j} \frac{\partial}{\partial \tau} \hat{\beta}_{i-1}(\tau)+\delta_{i, j+1} \frac{\partial}{\partial \tau} \sqrt{\frac{\alpha_{i-2}(\tau) \hat{\gamma}_{i-1}(\tau)}{d_{2}}}\right)_{i, j=1}^{N},
$$

where $\hat{\gamma}_{i}(\tau)=\gamma_{i}(\tau) /\left(\beta_{i}(\tau) \beta_{i-1}(\tau)\right), \hat{\beta}_{i-1}(\tau)=1 / \beta_{i-1}(\tau), i=1, \ldots, N$, is positive (negative) definite, then those zeros of $B_{1}(x ; \tau), \ldots, B_{N}(x ; \tau)$, which are greater than the positive real number $d_{2}$, are strictly decreasing (increasing) functions of $\tau, \tau \in$ $(p, q)$.

Theorem 2.3 in [8] provides a powerful tool for testing the monotonic behaviour of zeros of orthogonal polynomials. Since it is not easy to check if a matrix of a general type is positive definite, Ismail and Muldoon used some criteria for positive definiteness of a tridiagonal matrix in order to reformulate their Theorem 2.3 into Theorem 3.3 which gives sufficient conditions for monotonicity of zeros of orthogonal polynomials in terms of chain sequences. We shall do the same with our Theorem 2.

Recall that the sequence $\left\{c_{n}\right\}_{1}^{N}$ is known as a (finite) chain sequence or more precisely positive chain sequence (see [1]) if there exists a second sequence $\left\{g_{n}\right\}_{0}^{N}$ such that

$$
c_{n}=\left(1-g_{n-1}\right) g_{n}, \quad 1<n \leq N, \quad 0 \leq g_{0}<1 \text { and } 0<g_{n}<1, \quad 1 \leq n \leq N .
$$

If $0 \leq g_{n}<1,0 \leq n \leq N$, then we refer to $\left\{c_{n}\right\}_{1}^{N}$ as a (finite) semi positive chain sequence. Note that $g_{n}=0$ iff $c_{n}=0$. Hence a semi positive chain sequence is made up of positive chain sequences. For example, if $\left\{c_{n}\right\}_{1}^{N}$ is a semi positive chain sequence, where $c_{k}=0$ and $c_{n} \neq 0$ for $n=1, \cdots, k-1, k+1, \cdots, N$, then both $\left\{c_{n}\right\}_{1}^{k-1}$ and $\left\{c_{n}\right\}_{k+1}^{N}$ are positive chain sequences.

Corollary 1. Let $B_{k}(x ; \tau), \quad k=0,1, \ldots, N$ satisfy (1.3), where $\alpha_{k}(\tau), \beta_{k}(\tau)$ and $\gamma_{k}(\tau), \quad k=0, \ldots, N-1$, are positive and differentiable functions of $\tau$ for $\tau \in$ $(p, q)$. If $\partial \beta_{i}(\tau) / \partial \tau, \quad i=0, \ldots, N-1$, are all positive (negative) and

$$
d_{1}\left(\frac{\partial}{\partial \tau} \sqrt{\alpha_{i-1}(\tau) \gamma_{i}(\tau)}\right)^{2} /\left(\frac{\partial \beta_{i}(\tau)}{\partial \tau} \frac{\partial \beta_{i-1}(\tau)}{\partial \tau}\right) \quad i=1, \ldots, N-1
$$

is a semi positive chain sequence for any $\tau \in(p, q)$, then all the zeros of $B_{1}(x ; \tau), \ldots$, $B_{N}(x ; \tau)$, which are less than $d_{1}$, are strictly increasing (decreasing) functions of $\tau, \quad \tau \in(p, q)$.

In particular, these zeros increase (decrease) when $\tau$ increases if $\partial \beta_{i}(\tau) / \partial \tau, \quad i=$ $0, \ldots, N-1$, are positive (negative) and

$$
4 d_{1} \cos ^{2}(\pi /(N+1))\left(\frac{\partial}{\partial \tau} \sqrt{\alpha_{i-1}(\tau) \gamma_{i}(\tau)}\right)^{2} \leq \frac{\partial \beta_{i}(\tau)}{\partial \tau} \frac{\partial \beta_{i-1}(\tau)}{\partial \tau} \quad i=1, \ldots, N-1
$$

Corollary 2. Let $B_{k}(x ; \tau), \quad k=0,1, \ldots, N$ satisfy (1.3), where $\alpha_{k}(\tau), \beta_{k}(\tau)$ and $\gamma_{k}(\tau), \quad k=0, \ldots, N-1$, are positive and differentiable functions of $\tau$ for $\tau \in$ $(p, q)$. If $\partial \hat{\beta}_{i}(\tau) / \partial \tau, \quad i=0, \ldots, N-1$, are all positive (negative) and

$$
\left(\frac{\partial}{\partial \tau} \sqrt{\alpha_{i-1}(\tau) \hat{\gamma}_{i}(\tau)}\right)^{2} /\left(d_{2} \frac{\partial \hat{\beta}_{i}(\tau)}{\partial \tau} \frac{\partial \hat{\beta}_{i-1}(\tau)}{\partial \tau}\right) \quad i=1, \ldots, N-1,
$$


is a semi positive chain sequence for any $\tau \in(p, q)$, then those zeros of $B_{1}(x ; \tau), \ldots$, $B_{N}(x ; \tau)$, which are greater than $d_{2}$, are strictly decreasing (increasing) functions of $\tau, \quad \tau \in(p, q)$.

In particular, these zeros decrease (increase) when $\tau$ increases if $\partial \hat{\beta}_{i}(\tau) / \partial \tau, \quad i=$ $0, \ldots, N-1$, are positive (negative) and

$$
4 \cos ^{2}(\pi /(N+1))\left(\frac{\partial}{\partial \tau} \sqrt{\alpha_{i-1}(\tau) \hat{\gamma}_{i}(\tau)}\right)^{2} \leq d_{2} \frac{\partial \hat{\beta}_{i}(\tau)}{\partial \tau} \frac{\partial \hat{\beta}_{i-1}(\tau)}{\partial \tau} i=1, \ldots, N-1
$$

As it happens, not all the zeros of orthogonal polynomials increase (decrease) simultaneously when the parameter increases. A typical example is the behaviour of zeros of the ultraspherical polynomials, orthogonal on $(-1,1)$ with respect to $\omega(x ; \lambda)=$ $\left(1-x^{2}\right)^{\lambda-1 / 2}, \lambda>-1 / 2$. The positive zeros decrease when $\lambda$ increases, while, because of the symmetry, the negative zeros increase with $\lambda$. Then the question of the extreme zeros arises. Results concerning this question about orthogonal polynomials are given by Ismail $[5,6]$. A similar phenomenon appears with the zeros of orthogonal Laurent polynomials. The next theorem is analogous to that proven in $[5,6]$.

TheOREM 1.3. Let $B_{k}(x ; \tau), k=0,1, \ldots, N-1$, satisfy (1.3), where $\alpha_{k}(\tau), \beta_{k}(\tau)$ and $\gamma_{k}(\tau), \quad k=0, \ldots, N-1$, are all positive for $\tau \in(p, q)$.

(i) If $\alpha_{k-1}(\tau) \gamma_{k}(\tau), \quad \alpha_{k-1}(\tau) \gamma_{k}(\tau)+\beta_{k-1}(\tau), \quad k=1, \ldots, N-1$ and $\beta_{N-1}(\tau)$ are increasing (decreasing) functions of $\tau$ then the largest zero of $B_{N}(x ; \tau)$ is also an increasing (decreasing) function of $\tau$.

(ii) If $\alpha_{k-1}(\tau) \hat{\gamma}_{k}(\tau), \quad \alpha_{k-1}(\tau) \hat{\gamma}_{k}(\tau)+\hat{\beta}_{k-1}(\tau), \quad k=1, \ldots, N-1$ and $\hat{\beta}_{N-1}(\tau)$ are increasing (decreasing) functions of $\tau$ then the smallest zero of $B_{N}(x ; \tau)$ is a decreasing (increasing) function of $\tau$.

(iii) If $\alpha_{k-1}(\tau) \gamma_{k}(\tau), \quad \beta_{k}(\tau) \quad k=0, \ldots, N-1$, are increasing (decreasing) functions of $\tau$ then the largest zero of $B_{N}(x ; \tau)$ is an increasing (decreasing) function of $\tau$.

(iv) If $\beta_{k}(\tau) k=0, \ldots, N-1$, increase (decrease) and $\alpha_{k-1}(\tau) \gamma_{k}(\tau), k=0, \ldots, N-1$ decrease (increase) when $\tau$ increases then the smallest zero $B_{N}(x ; \tau)$ increases (decreases).

The proofs are given in the next section. Section 3 contains some illustrative examples.

\section{Proofs.}

Proof of Theorem 1. Jones and Thron [9] proved that for any strong distribution $\psi$ there exists a unique quadrature formula of the form $\int_{a}^{b} f(x) d \psi(x) \approx \sum_{i=1}^{n} A_{i} f\left(x_{i}\right)$ which is exact for every $f$ for which $x^{n} f(x) \in \pi_{2 n-1}\left(\pi_{k}\right.$ denotes the set of polynomials of degree $\leq k)$. Moreover, the nodes $x_{i}, i=1, \ldots, n$ of this quadrature rule coincide with the zeros of $B_{n}$ and its weights $A_{i}$ are positive. Hence

$$
\int_{a}^{b} f(x) \omega(x ; \tau) d \psi(x)=\sum_{i=1}^{n} A_{i}(\tau) f\left(\zeta_{i}(\tau)\right)
$$

whenever

$$
x^{n} f(x) \in \pi_{2 n-1} .
$$

Let $f$ be a fixed function which satisfies (2.2). Differentiate (2.1) with respect to $\tau$ and change the order of differentiation and integration. This is admissible because of 
the uniform convergence of the integrals (1.4). Thus

$$
\int_{a}^{b} f(x) \frac{\partial \omega(x ; \tau)}{\partial \tau} d \psi(x)=\sum_{i=1}^{n}\left\{A_{i}^{\prime}(\tau) f\left(\zeta_{i}(\tau)\right)+A_{i}(\tau) f^{\prime}\left(\zeta_{i}(\tau)\right) \zeta_{i}^{\prime}(\tau)\right\}
$$

Choose $f(x)=x^{-n} B_{n}^{2}(x ; \tau) /\left(x-\zeta_{k}(\tau)\right)$. Since $f$ satisfies (2.2) then (2.3) holds true for this. Furthermore, $f\left(\zeta_{i}(\tau)\right)=0$ and $f^{\prime}\left(\zeta_{i}(\tau)\right)=\left(\zeta_{k}(\tau)\right)^{-n}\left(B_{n}^{\prime}\left(\zeta_{k}(\tau) ; \tau\right)\right)^{2} \delta_{i k}$ for $i=1, \ldots, n$. Hence $(2.3)$ reduces to

$$
\int_{a}^{b} \frac{x^{-n} B_{n}^{2}(x ; \tau)}{x-\zeta_{k}(\tau)} \frac{\partial \omega(x ; \tau)}{\partial \tau} d \psi(x)=A_{k}(\tau)\left(\zeta_{k}(\tau)\right)^{-n}\left\{B_{n}^{\prime}\left(\zeta_{k}(\tau) ; \tau\right)\right\}^{2} \zeta_{k}^{\prime}(\tau)
$$

On the other hand $x^{-n} B_{n}(x ; \tau)$ is orthogonal on $(a, b)$ with respect to $d \psi(x ; \tau)$ to any polynomial of degree $n-1$. Hence

$$
\int_{a}^{b} x^{-n} B_{n}^{2}(x ; \tau) /\left(x-\zeta_{k}(\tau)\right) \omega(x ; \tau) d \psi(x)=0 .
$$

Subtract $\left\{\partial \omega\left(\zeta_{k}(\tau) ; \tau\right) / \partial \tau\right\} / \omega\left(\zeta_{k}(\tau) ; \tau\right)$ times the latter equality from (2.4) to obtain

$$
\begin{aligned}
& \int_{a}^{b} \frac{x^{-n} B_{n}^{2}(x ; \tau)}{x-\zeta_{k}(\tau)}\left[\frac{\partial \omega(x ; \tau) / \partial \tau}{\omega(x ; \tau)}-\frac{\partial \omega\left(\zeta_{k}(\tau) ; \tau\right) / \partial \tau}{\omega\left(\zeta_{k}(\tau) ; \tau\right)}\right] d \psi(x ; \tau) \\
= & A_{k}(\tau) \zeta_{k}^{-n}(\tau)\left\{B_{n}^{\prime}\left(\zeta_{k}(\tau) ; \tau\right)\right\}^{2} \zeta_{k}^{\prime}(\tau) .
\end{aligned}
$$

The expression in the square brackets has the same (opposite) sign as $x-\zeta_{k}(\tau)$ if $\partial \ln \omega(x ; \tau) / \partial \tau$ increases (decreases) in $x \in(a, b)$. Thus the integrand on the left-hand side of $(2.5)$ is positive (negative) if the logarithmic derivative with respect to $\tau$ of $\omega(x, \tau)$ increases (decreases) as a function of $x$ in $(a, b)$. This completes the proof of Theorem 1.

For the proof of Theorem 2 we need a matrix representation of L-orthogonal polynomials. We shall prove that the zeros of $B_{n}$ are solutions of a generalized eigenvalue problem. For any positive integer $N$ define the $n \times n$ matrices $A_{N}$ and $D_{N}$,

$$
\begin{gathered}
A_{N}(\tau)=\left(\begin{array}{cccccc}
0 & \alpha_{0}(\tau) & 0 & \cdots & 0 & 0 \\
\gamma_{1}(\tau) & 0 & \alpha_{1}(\tau) & \cdots & 0 & 0 \\
\vdots & \vdots & \vdots & & \vdots & \vdots \\
0 & 0 & 0 & \cdots & 0 & \alpha_{N-2}(\tau) \\
0 & 0 & 0 & \cdots & \gamma_{N-1}(\tau) & 0
\end{array}\right) \\
D_{N}(\tau)=\operatorname{diag}\left(\beta_{0}(\tau), \beta_{1}(\tau), \cdots, \beta_{N-1}(\tau)\right)
\end{gathered}
$$

Let $Q_{n}(x ; \tau)=x^{-n / 2} B_{n}(x ; \tau), \quad n=0,1, \ldots$ Then $Q_{n}$ satisfy the recurrence relation

$$
\begin{aligned}
& x^{1 / 2} Q_{n}(x ; \tau) \\
= & \alpha_{n}(\tau) Q_{n+1}(x ; \tau)+\beta_{n}(\tau) x^{-1 / 2} Q_{n}(x ; \tau)+\gamma_{n}(\tau) Q_{n-1}(x ; \tau), \quad n \geq 0
\end{aligned}
$$

with $Q_{-1}(x ; \tau)=0, \quad Q_{0}(x ; \tau)=1$, where $\alpha_{n}(\tau), \beta_{n}(\tau)$ and $\gamma_{n}(\tau)$ are positive for any $\tau \in(p, q)$. For any positive integer $N$ and real $x \in(a, b)$ and $\tau \in(p, q)$, define the $N$-dimensional vector-column

$$
y_{N}(x ; \tau)=\left(Q_{0}(x ; \tau), \ldots, Q_{N-1}(x ; \tau)\right)^{T} .
$$


The recurrence relation (2.6) yields the vector identity

$$
-\alpha_{N-1}\left(0, \ldots, 0, Q_{N}(x ; \tau)\right)^{T}=\left(A_{N}(\tau)+x^{-1 / 2} D_{N}(\tau)-x^{1 / 2} I\right) y_{N}(x ; \tau)
$$

Therefore $\alpha_{0} \ldots \alpha_{N-1} Q_{N}(x ; \tau)$ can be represented in determinant form as

$$
\left|\begin{array}{cccccc}
\sqrt{x}-\beta_{0} / \sqrt{x} & \alpha_{0} & 0 & \cdots & 0 & 0 \\
\gamma_{1} & \sqrt{x}-\beta_{1} / \sqrt{x} & \alpha_{1} & \cdots & 0 & 0 \\
\vdots & \vdots & \vdots & & \vdots & \vdots \\
0 & 0 & 0 & \cdots & \sqrt{x}-\beta_{N-2} / \sqrt{x} & \alpha_{N-2} \\
0 & 0 & 0 & \cdots & \gamma_{N-1} & \sqrt{x}-\beta_{N-1} / \sqrt{x}
\end{array}\right| .
$$

Renormalizing $Q_{n}, n=1,2, \ldots$ by $\tilde{Q}_{n}(x)=\left(\alpha_{0} / \gamma_{1}\right)^{1 / 2} \ldots\left(\alpha_{n-1} / \gamma_{n}\right)^{1 / 2} Q_{n}(x)$ we obtain the recurrence relation

$$
x^{1 / 2} \tilde{Q}_{n}(x)=\theta_{n+1} \tilde{Q}_{n+1}(x)+\beta_{n} x^{-1 / 2} \tilde{Q}_{n}(x)+\theta_{n} \tilde{Q}_{n-1}(x),
$$

where $\theta_{n}=\sqrt{\alpha_{n-1} \gamma_{n}}$. If $\tilde{A}_{n}(\tau)$ is the Hermitian matrix

$$
\tilde{A}_{N}(\tau)=\left(\begin{array}{cccccc}
0 & \theta_{1}(\tau) & 0 & \cdots & 0 & 0 \\
\theta_{1}(\tau) & 0 & \theta_{2}(\tau) & \cdots & 0 & 0 \\
\vdots & \vdots & \vdots & & \vdots & \vdots \\
0 & 0 & 0 & \cdots & 0 & \theta_{N-1}(\tau) \\
0 & 0 & 0 & \cdots & \theta_{N-1}(\tau) & 0
\end{array}\right)
$$

and

$$
\tilde{y}_{N}(x ; \tau):=\left(\tilde{Q}_{0}(x ; \tau), \ldots, \tilde{Q}_{N-1}(x ; \tau)\right)^{T}
$$

then (2.7) and (2.8) are transformed to

$$
-\theta_{N}\left(0, \ldots, 0, \tilde{Q}_{N}(x ; \tau)\right)^{T}=\left(\tilde{A}_{N}(\tau)+x^{-1 / 2} D_{N}(\tau)-x^{1 / 2} I\right) \tilde{y}_{N}(x ; \tau)
$$

and

$$
\begin{aligned}
& \theta_{1} \ldots \theta_{N} \tilde{Q}_{N}(x, \tau)= \\
& \left|\begin{array}{cccccc}
\sqrt{x}-\beta_{0} / \sqrt{x} & \theta_{1} & 0 & \cdots & 0 & 0 \\
\theta_{1} & \sqrt{x}-\beta_{1} / \sqrt{x} & \theta_{2} & \cdots & 0 & 0 \\
\vdots & \vdots & \vdots & & \vdots & \vdots \\
0 & 0 & 0 & \cdots & \sqrt{x}-\beta_{N-2} / \sqrt{x} & \theta_{N-1} \\
0 & 0 & 0 & \cdots & \theta_{N-1} & \sqrt{x}-\beta_{N-1} / \sqrt{x}
\end{array}\right|,
\end{aligned}
$$

respectively.

Proof of Theorem 2. It follows immediately from (2.9) that the zeros $\zeta_{k}$ of $B_{N}$ are the solutions of the generalized eigenvalue problem

$$
\left(\tilde{A}_{N}(\tau)+x^{-1 / 2} D_{N}(\tau)-x^{1 / 2} I\right) y=0
$$


and an eigenvector which corresponds to $\zeta_{k}$ is $y_{N k}(\tau):=\tilde{y}_{N}\left(\zeta_{k}(\tau) ; \tau\right)$. In the remainder of the proof we shall actually prove the Hellman-Feynman theorem (see $[6,12])$ for the problem (2.11). Substitute $x=\zeta_{k}(\tau)$ in (2.11) and multiply the resulting equality by $y_{N k}$. We obtain consecutively

$$
\left(\tilde{A}_{N}(\tau)+\zeta_{k}^{-1 / 2}(\tau) D_{N}(\tau)-\zeta_{k}^{1 / 2}(\tau) I\right) y_{N k}(\tau)=0
$$

and

$$
\left(y_{N k}, \tilde{A}_{N} y_{N k}\right)+\zeta_{k}^{-1 / 2}\left(y_{N k}, D_{N} y_{N k}\right)-\zeta_{k}^{1 / 2}\left(y_{N k}, y_{N k}\right)=0
$$

where (.,.) is a scalar product in Euclidian space $\mathbb{R}^{N}$. Recall that the zeros of $B_{N}$, and thus the solutions of (2.13) are distinct. By the implicit function theorem $\zeta_{k}$ is differentiable and its derivative can be obtained by differentiating (2.13),

$$
\begin{aligned}
& \left(y_{N k}^{\prime}, \tilde{A}_{N} y_{N k}\right)+\left(y_{N k}, \tilde{A}_{N}^{\prime} y_{N k}\right)+\left(y_{N k}, \tilde{A}_{N} y_{N k}^{\prime}\right) \\
& +\zeta_{k}^{-1 / 2}\left\{\left(y_{N k}^{\prime}, D_{N} y_{N k}\right)+\left(y_{N k}, D_{N}^{\prime} y_{N k}\right)+\left(y_{N k}, D_{N} y_{N k}^{\prime}\right)\right\} \\
& -2 \zeta_{k}^{1 / 2}\left(y_{N k}^{\prime}, y_{N k}\right)-\frac{1}{2}\left\{\zeta_{k}^{-3 / 2}\left(y_{N k}, D_{N} y_{N k}\right)+\zeta_{k}^{-1 / 2}\left(y_{N k}, y_{N k}\right)\right\} \zeta_{k}^{\prime}=0 .
\end{aligned}
$$

Here the prime means component-wise derivative with respect to $\tau$. Since $\tilde{A}_{N}$ and $D_{N}$ are Hermitian matrices they are self-adjoint operators in the Euclidian space $\mathbb{R}^{N}$. Thus $\left(x, \tilde{A}_{N} y\right)=\left(y, \tilde{A}_{N} x\right)$ and $\left(x, D_{N} y\right)=\left(y, D_{N} x\right)$ for any two $n$-dimensional vectors $x$ and $y$. On using this fact and (2.12) we obtain

$$
\begin{aligned}
& \left(y_{N k}^{\prime}, \tilde{A}_{N} y_{N k}\right)+\left(y_{N k}, \tilde{A}_{N} y_{N k}^{\prime}\right)+\zeta_{k}^{-1 / 2}\left\{\left(y_{N k}^{\prime}, D_{N} y_{N k}\right)+\left(y_{N k}, D_{N} y_{N k}^{\prime}\right)\right\} \\
& -2 \zeta_{k}^{1 / 2}\left(y_{N k}^{\prime}, y_{N k}\right) \\
= & 2\left(y_{N k}^{\prime},\left(\tilde{A}_{N}+\zeta_{k}^{-1 / 2} D_{N}-\zeta_{k}^{1 / 2} I\right) y_{N k}\right)=0 .
\end{aligned}
$$

Hence

$$
\begin{aligned}
& \frac{1}{2}\left\{\left(y_{N k}, D_{N} y_{N k}\right)+\zeta_{k}\left(y_{N k}, y_{N k}\right)\right\} \zeta_{k}^{-3 / 2} \zeta_{k}^{\prime} \\
= & \left(y_{N k},\left(\tilde{A}_{N}^{\prime}+\zeta_{k}^{-1 / 2} D_{N}^{\prime}\right) y_{N k}\right) \\
= & \left(\zeta_{k}^{-1 / 2}-d_{1}^{-1 / 2}\right)\left(y_{N k}, D_{N}^{\prime} y_{N k}\right)+\left(y_{N k},\left(\tilde{A}_{N}^{\prime}+d_{1}^{-1 / 2} D_{N}^{\prime}\right) y_{N k}\right) .
\end{aligned}
$$

Since $D_{N}$ is a diagonal positive matrix and $\zeta_{k}>0$ then $\zeta_{k}^{\prime}$ is certainly positive (negative) if $\tilde{A}_{N}^{\prime}+d_{1}^{-1 / 2} D_{N}^{\prime}$ is positive (negative) definite and $\zeta_{k}^{-1 / 2}-d_{1}^{-1 / 2}>0$. This completes the proof of statement (i).

Now the proof of statement (ii) of Theorem 2 follows from the observation that the polynomials $\hat{B}_{n}(x, \tau)=(-1)^{n}\left(\beta_{0}(\tau) \beta_{1}(\tau) \ldots \beta_{n-1}(\tau)\right)^{-1} x^{n} B_{n}(1 / x, \tau)$ satisfy the recurrence relation

$$
\hat{B}_{n}(x, \tau)=\alpha_{n}(\tau) \hat{B}_{n}(x, \tau)+\hat{\beta}_{n}(\tau) \hat{B}_{n}(x, \tau)+\hat{\gamma}_{n}(\tau) \hat{B}_{n}(x, \tau), \quad n \geq 0,
$$

with $\hat{B}_{-1}=0, \hat{B}_{0}=1$ and $\beta_{-1}=1$.

For the proofs of the corollaries we need to formulate two known results. The first one is the so-called Wall and Wetzel theorem (see [17]). by

Theorem A. Let $A=\left[a_{i, j}\right]$ be a symmetric $(N \times N)$ tridiagonal matrix, given

$$
a_{i, j}=\alpha_{i} \delta_{i, j-1}+\beta_{i} \delta_{i, j}+\alpha_{i-1} \delta_{i, j+1}, \quad 1 \leq i, j \leq N
$$


Then $A$ is positive definite if and only if $\beta_{n}>0, n=1,2, \cdots, N$ and that there exist real numbers $g_{n}, 0 \leq n \leq N$, where $g_{0}=0,0 \leq g_{n}<1, n=1,2, \cdots, N$, such that

$$
\frac{\alpha_{n}^{2}}{\beta_{n} \beta_{n+1}}=\left(1-g_{n-1}\right) g_{n}, \quad n=1,2, \cdots, N-1 .
$$

Next theorem is due to Ismail and $\mathrm{Li}[7]$.

THEOREM B. The constant sequence $\{c\}_{1}^{N}$ is a finite positive chain sequence if and only if

$$
0<c<\left[4 \cos ^{2}(\pi /(N+2)]^{-1}\right.
$$

The first statement of Corollary 1 is a consequence of statement (i) of Theorem 2 and the Wall and Wetzel theorem. Note that the latter states that $A$ is positive definite if and only if $\left\{\frac{\alpha_{n}^{2}}{\beta_{n} \beta_{n+1}}\right\}_{1}^{N-1}$ is a semi positive chain sequence. The second statement follows from the comparison test for the chain sequences (see Theorem 5.7 in [1]) with the largest finite constant chain sequence given by Ismail and $\mathrm{Li}$ [7] (see Theorem B above). Similarly, statement (ii) of Theorem 2 leads to Corollary 2.

Proof of Theorem 3. Bearing in mind the definition of $y_{N k}$ and (2.14) we conclude

$$
\begin{aligned}
& \operatorname{sign} \zeta_{k}^{\prime} \\
= & \operatorname{sign} \sum_{i=0}^{N-1}\left[\left(\sqrt{\alpha_{i} \gamma_{i+1}}\right)^{\prime} \tilde{Q}_{i+1}\left(\zeta_{k}\right)+\zeta_{k}^{-1 / 2} \beta_{i}^{\prime} \tilde{Q}_{i}\left(\zeta_{k}\right)+\left(\sqrt{\alpha_{i-1} \gamma_{i}}\right)^{\prime} \tilde{Q}_{i-1}\left(\zeta_{k}\right)\right] \tilde{Q}_{i}\left(\zeta_{k}\right) .
\end{aligned}
$$

Recall that the zeros of the orthogonal Laurent polynomials are positive and distinct. Moreover, their leading coefficients are positive because they satisfy (1.3) with positive $\alpha_{n}$. Hence for the largest zero $\zeta_{1}$ of $B_{N}$ we have

$$
\tilde{Q}_{i}\left(\zeta_{1}\right)>0, \quad i=0, \ldots, N-1 .
$$

For the smallest zero $\zeta_{N}$ of $B_{N}$ we obtain

$$
\operatorname{sign} \tilde{Q}_{i}\left(\zeta_{N}\right)=(-1)^{i}, \quad i=0, \ldots, N-1 .
$$

Now statement (iii) of the theorem follows immediately from (2.15) and (2.16). Statement (iv) is a consequence of (2.15) and (2.17).

While the idea of the proof of (iii) and (iv) is applicable also for the orthogonal polynomials and it is essentially due to Ismail $[5,6]$, the proof of (i) we shall give now relies on a specific property of orthogonal L-polynomials. Sri Ranga and Andrade [14] proved that if $B_{n}$ satisfy the recurrence relation (1.3), then the zeros of $B_{N}$ are the eigenvalues of the upper Hessenberg matrix

$$
H_{N}=\left(\begin{array}{cccccc}
\alpha_{0} \gamma_{1}+\beta_{0} & \alpha_{1} \gamma_{2}+\beta_{1} & \alpha_{2} \gamma_{3}+\beta_{2} & \cdots & \alpha_{N-2} \gamma_{N-1}+\beta_{N-2} & \beta_{N-1} \\
\alpha_{0} \gamma_{1} & \alpha_{1} \gamma_{2}+\beta_{1} & \alpha_{2} \gamma_{3}+\beta_{2} & \cdots & \alpha_{N-2} \gamma_{N-1}+\beta_{N-2} & \beta_{N-1} \\
0 & \alpha_{1} \gamma_{2} & \alpha_{2} \gamma_{3}+\beta_{2} & \cdots & \alpha_{N-2} \gamma_{N-1}+\beta_{N-2} & \beta_{N-1} \\
\vdots & \vdots & \vdots & \vdots & \vdots & \vdots \\
0 & 0 & 0 & \cdots & \alpha_{N-2} \gamma_{N-1}+\beta_{N-2} & \beta_{N-1} \\
0 & 0 & 0 & \cdots & \alpha_{N-2} \gamma_{N-1} & \beta_{N-1}
\end{array}\right) .
$$


Actually, the result in [14] is stated and proved for monic Laurent polynomials and (2.18) follows by proper renormalization. Recall that the Perron-Frobenius theorem (see Theorem 8.4.5 in Horn and Johnson [4]) asserts that if a matrix with nonnegative elements majorises in the componentwise sense another matrix with nonnegative elements then the largest eigenvalue of the first matrix is not less than the largest eigenvalue of the second matrix. Now the Perron-Frobenius theorem and (2.18) demonstrate the truth of assertion (i) of the theorem.

The proof of (ii) follows the same way from the recurrence relation of the polynomials $\hat{B}_{n}$.

3. Monotonicity of Laguerre and Jacobi L-orthogonal polynomials. In this section we shall apply the ongoing results to investigate the monotonic behaviour of zeros of some sets of L-orthogonal polynomials which may be considered as Lanalogs of Laguerre and Jacobi polynomials. First we consider the orthogonal Laurent polynomials $H_{n}^{(\alpha, \beta)}$ associated with the weight function

$$
\omega(x ; \alpha, \beta)=x^{-1 / 2} \exp \left(-\frac{x+\beta^{2} / x}{4 \alpha}\right), \quad x \in(0, \infty),
$$

where $\alpha$ and $\beta$ are positive (see [13, Example 3]). Since

$$
\frac{\partial \ln \omega(x ; \alpha, \beta)}{\partial \beta}=-\frac{\beta}{2 \alpha x}
$$

is an increasing function of $x, x \in(0, \infty)$, then by Theorem 1 for any $n>0$ all the zeros of $H_{n}^{(\alpha, \beta)}$ increase with $\beta$. The matrices $D_{N}$ and $\tilde{A}_{N}$ associated with these L-orthogonal polynomials are: $D_{N}=\beta I$ and the elements of $\tilde{A}_{N}$ are $\theta_{j}=\sqrt{2 \alpha j}$. Hence $\frac{\partial}{\partial \beta}\left(\tilde{A}_{N}+d^{-1 / 2} D_{N}\right)=d^{-1 / 2} I$ is positive definite for all $d>0$. Then the increase of the zeros of $H_{n}^{(\alpha, \beta)}$ as functions of $\beta$ follows also from Theorem 2. Since $\frac{\partial}{\partial \alpha}\left(\alpha_{i-1} \gamma_{i}\right)=2 i>0$ for $i=1,2, \ldots$, the largest zero of $H_{n}^{(\alpha, \beta)}$ is also an increasing function of $\alpha$.

Hendriksen and van Rossum [3] considered the monic polynomials

$$
P_{n}^{(a, c)}(x):=\frac{(c)_{n}}{(1-a)_{n}}{ }_{2} F_{1}(-n, 1-a ; 1-c-n ; x)
$$

where $(c)_{n}$ is the Pochhammer symbol and ${ }_{2} F_{1}$ is the hypergeometric function. If $P_{-1}^{(a, c)}(x)=0$ then $P_{n}^{(a, c)}(x)$ satisfy the recurrence relation (see [2])

$$
P_{n}^{(a, c)}(x)=\left(x-\frac{c+n-1}{a-n}\right) P_{n-1}^{(a, c)}(x)-\frac{(n-1)(c+n-a-1)}{(a-n)(a+1-n)} x P_{n-2}^{(a, c)}(x), \quad n \geq 1 .
$$

It is clear that there are no values of $a$ and $c$ for which the coefficients $\beta_{n-1}=$ $(c+n-1) /(a-n), n \geq 1$ and $\gamma_{n-1}=(n-1)(c+n-a-1) /((a-n)(a+1-n))$, $n \geq 2$ are positive for every positive integer $n$. However, if

$$
\text { 1) } c>a>N, \quad \text { or } \quad 2) a<c<1-N \text {, }
$$

then the coefficients $\beta_{0}$ and $\beta_{n}, \gamma_{n}, n=1, \ldots, N-1$ are positive. In these cases we can apply Theorem 2 and Corollary 1 . We shall use Corollary 1 to investigate the monotonicity of the zeros of $P_{n}^{(a, c)}$ with respect to $c$. Since $\partial \beta_{n} / \partial c=(a-n-1)^{-1}$ 
and $\partial \sqrt{\gamma_{n}} / \partial c=(1 / 2) n^{1 / 2}\{(a-n)(a-n-1)(c-a+n)\}^{-1 / 2}$ then the zeros of $P_{n}^{(a, c)}, n=1, \ldots, N$, which are smaller than $\cos ^{-2}\left(\frac{\pi}{N+1}\right)$, are certainly increasing functions of $c$ if the parameters $a$ and $c$ satisfy 1). If $a$ and $c$ satisfy 2$)$ then the zeros of $P_{n}^{(a, c)}, n=1, \ldots, N$, which lie in the interval $\left(0, \cos ^{-2}\left(\frac{\pi}{N+1}\right)\right)$, are decreasing functions of $c$.

The last example concerns largest zeros of the ultraspherical L-polynomials $B_{n}^{\lambda}$ introduced in Example 2 in [13]. Since the orthogonal L-polynomials in [13] are obtained from symmetric orthogonal polynomials by a transformation which makes the ordinary central symmetry an inversion symmetry, it is clear that if the positive zeros of the symmetric orthogonal polynomials increase (decrease) then half of the zeros of the orthogonal L-polynomials will increase and the other half will decrease. It is shown in [13] that the ultraspherical polynomials are transformed to L-orthogonal polynomials which satisfy $(1.2)$ with $d \psi(x)=x^{-\lambda}(b-x)^{\lambda-1 / 2}(x-a)^{\lambda-1 / 2} d x$, where $\sqrt{b}=\sqrt{\alpha+\beta}+\sqrt{\alpha}, \sqrt{a}=\sqrt{\alpha+\beta}-\sqrt{\alpha}, \alpha, \beta>0$ and the coefficients in the recurrence relation (1.3) are given by $\alpha_{n}=1, \beta_{n}=\beta$ and $\gamma_{n}=\alpha n(n+2 \lambda-1) /((n+\lambda)(n+\lambda))$. By Theorem 2 all the zeros of $B_{n}^{\lambda}$ are increasing functions of $\beta$. Furthemore, since $\partial \gamma_{n} / \partial \alpha>0$ and

$$
-n \alpha(n+\lambda)^{2}(n+\lambda-1)^{2} \gamma_{n}^{\prime}(\lambda)=2 \lambda^{2}+(2 n-1) \lambda+1
$$

then the largest zeros of $B_{n}^{\lambda}$ are increasing functions of $\alpha$ and decreasing functions of $\lambda, \lambda \geq 0$.

\section{REFERENCES}

[1] T. S. Chimara, An Introduction to Orthogonal Polynomials, Gordon and Breach, New York, 1978.

[2] E. Hendriksen, A weight function for the associated Jacobi-Laurent polynomials, J. Comput. Appl. Math., 33 (1990), pp. 171-180.

[3] E. Hendriksen and H. van Rossum, Orthogonal Laurent polynomials, Nederl. Acad. Wetensch. Proc. Ser. A, 89 (1); also: Indag. Math., 48 (1) (1986), pp. 17-36.

[4] R. A. Horn and C. R. Johnson, Matrix Analysis, Cambridge University Press, Cambridge, 1990.

[5] M. E. H. Ismail, The variation of zeros of certain orthogonal polynomials, Adv. Appl. Math., 8 (1987), pp. 111-118.

[6] M. E. H. Ismail, Monotonicity of zeros of orthogonal polynomials, in: "q-Series and Partitions," (D.Stanton, Ed.), pp. 177-190, Springer-Verlag, New York, 1989.

[7] M. E. H. Ismail AND X. LI, Bounds on the extreme zeros of orthogonal polynomials, Proc. Amer. Math. Soc., 115 (1992), pp. 131-140.

[8] M. E. H. Ismail And M. E. Muldoon, A discrete approach to monotonicity of zeros of orthogonal polynomials, Trans. Amer. Math. Soc., 323 (1991), pp. 65-78.

[9] W. B. Jones And W. J. Thron, Orthogonal Laurent polynomials and Gaussian quadrature, in: "Quantum Mechanics in Mathematics, Chemistry, and Physics," (K. E. Gustafson and W. P. Reinhardt, Eds.), pp. 449-455, Plenum Press, New York, 1981.

[10] W. B. Jones, W. J. Thron And H. WaAdeland, A strong Stieltjes moment problem, Trans. Amer. Math. Soc., 261 (1980), pp. 503-528.

[11] A. Markov, Sur les racines de certains équations (second note), Math. Ann., 27 (1886), pp. $177-182$.

[12] M. E. Muldoon, Properties of zeros of orthogonal polynomials and related functions, J. Comput. Appl. Math., 48 (1993), pp. 167-186.

[13] A. SRI RANGA, Symmetric orthogonal polynomials and the associated orthogonal L-polynomials, Proc. Amer. Math. Soc., 123 (1995), pp. 3135-3141.

[14] A. SRi RAnga And E. X. L. DE Andrade, Zeros of polynomials which satisfy a certain three term recurrence relation, Comm. Anal. Theory Cont. Frac., 1 (1992), pp. 61-65.

[15] T. J. Stieltues, Sur les racines de l'équation $X_{n}=0$, Acta Math., 9 (1886), pp. 385-400. 
[16] G. SzegŐ, Orthogonal Polynomials, 4th ed., Amer. Math. Soc. Coll. Publ., Vol. 23, Providence, RI, 1975.

[17] H. S. WALL AND M. WETZEL, Quadratic forms and convergence regions of continued fractions, Duke Math. J., 11 (1944), pp. 89-102. 
